\title{
ESTUDO DO PRÉ-TRATAMENTO ÁCIDO DA PALMA FORRAGEIRA PARA A PRODUÇÃO DE ETANOL DE SEGUNDA GERAÇÃOO
}

\author{
D. P. C. ANTUNES ${ }^{1}$, M. C. S. SANTOS ${ }^{1}$, E. L. S. SOARES ${ }^{1}$, \\ K. L. BARBOSA ${ }^{1}$, R. C. VIEIRA ${ }^{1}$, V. L. VIANA ${ }^{1}$, R. M. R. G. ALMEIDA ${ }^{1}$ \\ ${ }^{1}$ Universidade Federal de Alagoas, Centro de Tecnologia, Departamento de Engenharia Química \\ E-mail para contato: renatarosas_ufal@hotmail.com
}

\begin{abstract}
RESUMO - A palma forrageira é uma das principais alternativas para alimentação de caprinos, ovinos e bovinos no Semiárido brasileiro. Atualmente as variedades mais plantadas no Nordeste brasileiro são as palmas do tipo Opuntia (OP) e Nopalea (NP). De acordo com a literatura, a palma possui cerca de $3 \%$ de hemicelulose, $21 \%$ de celulose e $4 \%$ de lignina, podendo ser utilizada para produção de etanol lignocelulósico. $\mathrm{O}$ objetivo desse trabalho foi estudar o pré-tratamento dessas duas variedades de palma com diferentes ácidos $\left(\mathrm{H}_{2} \mathrm{SO}_{4}, \mathrm{HCl}, \mathrm{HNO}_{3}\right.$ e $\left.\mathrm{H}_{3} \mathrm{PO}_{4}\right)$ em duas diferentes concentrações de ácido (0,5 e 1\%) e variando- se o tempo do processo (15 e $30 \mathrm{~min}$ ). As repostas avaliadas foram: rendimento mássico, Brix, açúcares redutores (AR) e açúcares redutores totais (ART). Antes do pré-tratamento, as palmas foram secas e trituradas, em seguida pré-tratada com ácido na proporção de sólido-líquido de 1:20, no reator do tipo auto-clave, a uma temperatura de $120{ }^{\circ} \mathrm{C}$, e 1 atm de pressão. Devido à baixa quantidade de lignina presente no material todos os prétratamentos realizados obtiveram valores elevados de AR, ART e Brix, sendo os maiores valores encontrados com o pré-tratamento da variedade OP com ácido sulfúrico 0,5\% (15,66 g/L de AR, 35,29 g/L de ART, 4,33 Brix e rendimento mássico de $61 \%$ ).
\end{abstract}

\section{INTRODUÇÃO}

Segundo Santos (2011) a palma é uma cultura originária do México, sendo atualmente cultivada em todo o mundo. Provavelmente foi introduzida no país durante o período de colonização para a produção da cochonilha do carmim. Em 1893, Barbosa Rodrigues recomendou o uso de palma como forrageira para alimentação do gado nas épocas de seca (Menezes et al., 2005). A maior área de palma forrageira no Nordeste se concentra no agreste e sertão dos estados de Alagoas e Pernambuco. Segundo dados do IPA (2001), cultivos bem conduzidos de palma forrageira produzem uma biomassa superior a 150 toneladas de matéria verde/ha/ano (ou 15 toneladas de matéria seca/ha/ano), desde que se associem práticas agronômicas adequadas e variedades de elevado potencial produtivo. A palma forrageira é uma fonte alimentar que pode viabilizar a produção animal no Semiárido do Nordeste brasileiro, devido às suas características morfofisiológicas e nutricionais (Lira et al., 2006). 
As espécies Opuntia ficus-indica Mill. cvs. Gigante (OP) e Nopalea cochenillifera Salm Dyck cv. Miúda (NP) são as variedades mais plantadas na região Nordeste do Brasil, sendo que a espécie Miúda é plantada em larga escala no estado de Alagoas, enquanto que nos outros estados nordestinos predomina o plantio de cultivares de da variedade OP (Santos et al., 2010).

Estudos realizados por Tosto (2007) sobre a composição química da palma forrageira demonstram que ela possui cerca de 3,30\% de hemicelulose, $21,12 \%$ de celulose e apenas 4,62 $\%$ de lignina, além de possuir 77,89 \% de carboidratos totais, o que a torna uma fonte rica em açúcares e passível de ser fermentada.

A crescente preocupação com o desenvolvimento sustentável vem dando ênfase a busca por fontes alternativas de energia que possam competir e/ou substituir a utilização de combustíveis fósseis (Chen, et. al., 2006). Nesse sentido, faz-se necessário investir em tecnologias para melhoramento de culturas como da palma forrageira a fim de obter dados para produtividade e representação dessa biomassa e transformá-la em fonte de energia renovável como o etanol de segunda geração (Sánchez et al., 2012).

Sabe-se que para uma biomassa lignocelulósica produzir etanol $2 \mathrm{G}$ são necessários quatro processos fundamentais, são eles: Pré-tratamento, Hidrólise, Fermentação e Destilação. A maior dificuldade encontrada nesse processo é a barreira da lignina presente na maior parte dos materiais utilizados para este tipo de conversão, sendo de fundamental importância a etapa do pré-tratamento que irá solubilizar a lignina liberando celulose para o meio. De acordo com Wolf (2011), atualmente o pré-tratamento mais utilizado para este tipo de processo é o realizado por meio de ácidos diluídos, onde o material é pré-tratado em meio a uma solução ácida de baixa concentração. O trabalho teve como objetivo estudar o pré-tratamento ácido da palma forrageira, avaliando as espécies NP e OP, a partir de análises de açúcares redutores (AR), açúcares redutores totais (ART), Brix e rendimento mássico, com o intuito de produzir etanol a partir deste material.

\section{METODOLOGIA}

Todos os ensaios foram realizados no Laboratório de Tecnologia de Bebidas e Alimentos, localizado no Centro de Tecnologia da Universidade Federal de Alagoas.

\subsection{Higienização, secagem e trituração das palmas}

As espécies de palma OP e NP sofreram processo de sanitização, que consistiu em lavagem com 100 ppm de água sanitária por 15 minutos. Após esse tempo, foram lavadas em água corrente. Em seguida, as palmas passaram por secagem em estufa $\left(\grave{a} 45^{\circ} \mathrm{C}\right)$. Após a secagem foram trituradas em um moinho de facas tipo Willye. 


\subsection{Pré-tratamento da palma forrageira}

Esta etapa consistiu em pré-tratar as espécies de palma com solução de ácido diluído variando-se a concentração de 0,5 e $1 \%$ de ácido, os ácidos utilizados no estudo do prétratamento foram ácido sulfúrico $\left(\mathrm{H}_{2} \mathrm{SO}_{4}\right)$, ácido fosfórico $\left(\mathrm{H}_{3} \mathrm{PO}_{4}\right)$, ácido clorídrico $(\mathrm{HCl}) \mathrm{e}$ ácido nítrico $\left(\mathrm{HNO}_{3}\right)$. Cerca de $1 \mathrm{~g}$ de material foi colocado em frasco do tipo Erlenmayer, levando-se em seguida para um reator do tipo autoclave a 1 atm e $120^{\circ} \mathrm{C}$, e uma proporção de sólido-líquido de 1:20, variando-se o tempo de reação de 15 e $30 \mathrm{~min}$, todo o experimento foi realizado em triplicata. Posteriormente, cada amostra foi filtrada, e foram realizadas análises de açúcares redutores (AR), açúcares redutores totais (ART) e Brix, na fração líquida, enquanto que a fração sólida foi secada para analisar o rendimento mássico obtido em cada condição estudada.

\subsection{Determinação de açúcares redutores}

Para este procedimento transfere-se $1,0 \mathrm{~mL}$ de amostra para um tubo e adiciona-se $5 \mathrm{~mL}$ de $\mathrm{H}_{2} \mathrm{SO}_{4}$ 1,5 M, levando-se a banho fervente por $20 \mathrm{~min}$ e agitando ocasionalmente para hidrolisar os polissacarídeos e outros açúcares não-redutores (ao menos a cada 5 min). Resfria-se e se adiciona, cuidadosamente, solução de $\mathrm{NaOH} 26 \%$ até neutralizar a amostra, completando-se em seguida o volume com água destilada de acordo com a diluição necessária.

Em seguida, realiza-se a reação de DNS, onde transfere-se $0,25 \mathrm{~mL}$ da amostra para um tubo de ensaio com tampa e adiciona $0,5 \mathrm{~mL}$ de água destilada. Após Verificar se o banho de água está fervendo, adiciona $0,25 \mathrm{~mL}$ do reagente de DNS e leva a amostra para banho fervente por 5min, permitindo que ocorra a reação entre a glicose e o DNS. Resfria-se o tubo em banho de gelo, ajuste o volume com água destilada para $5 \mathrm{~mL}$ (ou seja, adicione $4 \mathrm{~mL}$ de água) e lê-se em comprimento de onda de $540 \mathrm{~nm}$, tendo como padrão concentrações conhecidas de glicose. A partir da curva padrão de concentrações conhecidas de glicose tem-se a equação da reta que permitirá calcular a concentração de glicose em cada amostra a partir da equação 1, onde a diluição da amostra será dada pela equação 2.

$\mathrm{AR}(\mathrm{g} / \mathrm{L})=$ fator da curva padrão.Absorbância.diluição

Onde,

Diluição = Volume da amostra $(\mathrm{mL}) /($ Volume Total $)(\mathrm{mL})$

\subsection{Determinação de açúcares redutores totais}

Transfere-se 0,25 $\mathrm{mL}$ da amostra para um tubo de ensaio com tampa e adiciona $0,5 \mathrm{~mL}$ de água destilada. Verifica-se se o banho de água está fervendo. Se sim, adiciona $0,25 \mathrm{~mL}$ do reagente de DNS e leva-se a amostra para banho fervente por $5 \mathrm{~min}$, permitindo que ocorra a reação entre a glicose e o DNS. Resfria-se o tubo em banho de gelo, ajusta o volume com água 
destilada para $5 \mathrm{~mL}$ (ou seja, adicione $4 \mathrm{~mL}$ de água) e lê-se em absorbância a 540nm, tendo como padrão concentrações conhecidas de glicose. A partir da curva padrão de concentrações conhecidas de glicose tem-se a equação da reta que permitirá calcular a concentração de glicose em cada amostra a partir da equação 1, e em caso de diluição também usa-se a equação 2.

\subsection{Determinação do rendimento do pré-tratamento}

O rendimento é calculado a partir da diferença de massa entre o inicio e o final do prétratamento. Pesa-se a massa inicial antes de ser pré-tratada, após o pré-tratamento o material é filtrado em papel de filtro previamente pesado e é levado para uma estufa a uma temperatura de aproximadamente $40{ }^{\circ} \mathrm{C}$ até secagem completa do material e do filtro. Em seguida, pesa-se o filtro com o material, onde é feito o calculo do rendimento pela equação 3, onde a massa final é dada pela equação 4.

$$
\text { Rendimento }=\left[\mathrm{M}_{\text {inicial }}-\mathrm{M}_{\text {final }}\right] / \mathrm{M}_{\text {inicial }}
$$

Onde,

$$
\mathrm{M}_{\text {final }}=\text { Massa do material com o filtro }- \text { Massa do filtro }
$$

\subsection{Determinação do Brix}

O Brix foi determinado por meio do refratômetro digital que forneceu a leitura em grau Brix, servindo como base para comparação com a quantidade de açúcares totais presentes na amostra.

\section{RESULTADO E DUSCUSSÕES}

Todos os resultados reportados são descritos com o valor da média dos níveis em estudo, uma vez que as amostras foram realizadas em triplicata. A Tabela 1 apresenta os resultados do pré-tratamento obtidos para duas variedades de palma OP e NP com todos os ácidos utilizados na concentração de $0,5 \%$ durante 15 minutos.

Ao se observar os resultados da Tabela 1 em termos de AR, ART e Brix o ácido sulfúrico apresentou os melhores resultados, apesar de gerar o maior rendimento o que significa uma maior perda de massa nas duas espécies de palma estudadas. A Tabela 2 mostra os resultados obtidos para duas variedades de palma OP e NP com todos os ácidos utilizados na concentração de 0,5\% durante 30 minutos. 
Tabela 1 - Resultados do pré-tratamento para $0,5 \%$ de ácido e 15 minutos.

\begin{tabular}{|c|c|c|c|c|c|}
\hline Biomassa & Ácido & AR & ART & BRIX & Rend \% \\
\hline \multirow{4}{*}{ NP } & $\mathrm{H}_{3} \mathrm{PO}_{4}$ & $3,67 \pm 1,41$ & $25,64 \pm 1,81$ & $3,86 \pm 0,10$ & 55,00 \\
\cline { 2 - 6 } & $\mathrm{HCL}$ & $3,47 \pm 1,21$ & $22,24 \pm 4,68$ & $3,40 \pm 0,01$ & 52,33 \\
\cline { 2 - 6 } & $\mathrm{HNO}_{3}$ & $4,08 \pm 0,36$ & $28,57 \pm 1,37$ & $3,53 \pm 0,06$ & 58,33 \\
\cline { 2 - 6 } & $\mathrm{H}_{2} \mathrm{SO}_{4}$ & $14,54 \pm 1,84$ & $33,00 \pm 4,75$ & $5,00 \pm 0,92$ & 59,66 \\
\cline { 2 - 6 } & & & & & \\
\hline \multirow{4}{*}{ OP } & $\mathrm{H}_{3} \mathrm{PO}_{4}$ & $4,08 \pm 1,48$ & $29,95 \pm 5,89$ & $3,46 \pm 0,46$ & 48,00 \\
\cline { 2 - 6 } & $\mathrm{HCL}$ & $3,39 \pm 0,44$ & $24,77 \pm 3,45$ & $3,16 \pm 0,06$ & 51,00 \\
\cline { 2 - 6 } & $\mathrm{HNO}_{3}$ & $5,97 \pm 1,39$ & $33,86 \pm 1,77$ & $3,50 \pm 0,14$ & 55,66 \\
\cline { 2 - 6 } & $\mathrm{H}_{2} \mathrm{SO}_{4}$ & $15,66 \pm 4,02$ & $35,29 \pm 10,67$ & $4,33 \pm 0,21$ & 61,00 \\
\hline
\end{tabular}

Tabela 2 - Resultados do pré-tratamento para $0,5 \%$ de ácido e 30 minutos.

\begin{tabular}{|c|c|c|c|c|c|}
\hline Biomassa & Ácido & AR & ART & BRIX & Rend \% \\
\hline \multirow{4}{*}{ NP } & $\mathrm{H}_{3} \mathrm{PO}_{4}$ & $6,71 \pm 0,61$ & $16,79 \pm 0,62$ & $3,53 \pm 0,15$ & 61,56 \\
\cline { 2 - 6 } & $\mathrm{HCL}$ & $8,59 \pm 3,24$ & $18,49 \pm 0,87$ & $3,36 \pm 0,06$ & 58,80 \\
\cline { 2 - 6 } & $\mathrm{HNO}_{3}$ & $6,54 \pm 0,02$ & $18,11 \pm 0,62$ & $3,40 \pm 0,01$ & 60,69 \\
\cline { 2 - 6 } & $\mathrm{H}_{2} \mathrm{SO}_{4}$ & $6,43 \pm 0,12$ & $20,12 \pm 1,80$ & $4,06 \pm 0,11$ & 54,76 \\
\cline { 2 - 6 } & \multicolumn{5}{|c}{} \\
\hline \multirow{4}{*}{ OP } & $\mathrm{H}_{3} \mathrm{PO}_{4}$ & $6,46 \pm 0,26$ & $21,19 \pm 0,24$ & $3,66 \pm 0,11$ & 70,26 \\
\cline { 2 - 6 } & $\mathrm{HCL}^{\mid}$ & $5,86 \pm 0,96$ & $22,83 \pm 0,28$ & $3,60 \pm 0,01$ & 60,86 \\
\cline { 2 - 6 } & $\mathrm{HNO}_{3}$ & $7,07 \pm 0,91$ & $23,76 \pm 0,59$ & $3,66 \pm 0,01$ & 69,86 \\
\cline { 2 - 6 } & $\mathrm{H}_{2} \mathrm{SO}_{4}$ & $5,66 \pm 0,27$ & $24,24 \pm 0,54$ & $4,26 \pm 0,06$ & 70,43 \\
\hline
\end{tabular}

Na Tabela 2 pode-se perceber que aumentando o tempo do processo de pré-tratamento a diferença entre os valores de AR, ART e Brix para os diferentes tipos de ácido não se mostrou significativa. Comparando-se a Tabela 1 com a Tabela 2, observa-se que o aumento do tempo para o pré-tratamento diminuiu a quantidade de ART liberada no meio independente do tipo de ácido utilizado, mesmo que os valores de AR estejam maiores para 30 minutos do que em 15 minutos. O que provavelmente pode ter ocorrido é uma degradação de parte dos açúcares presentes no meio devido ao maior tempo de contato com os ácidos. A Tabela 3 apresenta os resultados obtidos para o pré-tratamento com concentração de ácido de $1 \%$ e tempo de 15 minutos para as duas espécies de palma estudadas.

Na Tabela 3 é possível observar que o ácido sulfúrico liberou mais açúcares que os demais ácidos apenas para espécie NP, enquanto que a espécie OP o valor mais alto de ART foi obtido utilizando-se o ácido fosfórico. Porém, comparando-se a Tabela 3 com a Tabela 1 observa-se que a menor concentração $(0,5 \%)$ para todos os tipos de ácido liberou mais açúcares em termos de ART tanto para espécie NP quanto para OP, apesar dos rendimentos mássicos estarem muito parecidos. Esses resultados corroboram com os de Santos (2014) que verificou que menores concentrações de ácido sulfúrico foram mais eficientes no pré-tratamento de palha e 
sabugo de milho.Os dados de pré-tratamento para concentração de $1 \%$ de ácido e 30 minutos de reação estão mostrados na Tabela 4 .

Tabela 3 - Resultados do pré-tratamento para 1,0\% de ácido e 15 minutos.

\begin{tabular}{|c|c|c|c|c|c|}
\hline Biomassa & Ácido & AR & ART & BRIX & Rend \% \\
\hline \multirow{4}{*}{ NP } & $\mathrm{H}_{3} \mathrm{PO}_{4}$ & $3,26 \pm 0,45$ & $14,50 \pm 0,46$ & $4,15 \pm 0,07$ & 52,35 \\
\cline { 2 - 6 } & $\mathrm{HCL}$ & $1,96 \pm 0,67$ & $17,79 \pm 1,14$ & $3,33 \pm 0,11$ & 51,60 \\
\cline { 2 - 6 } & $\mathrm{HNO}_{3}$ & $8,11 \pm 1,93$ & $19,45 \pm 0,64$ & $3,66 \pm 0,06$ & 61,16 \\
\cline { 2 - 6 } & $\mathrm{H}_{2} \mathrm{SO}_{4}$ & $11,73 \pm 1,43$ & $23,30 \pm 2,26$ & $4,44 \pm 0,43$ & 59,60 \\
\cline { 2 - 6 } & \multicolumn{5}{|c|}{} \\
\hline \multirow{4}{*}{ OP } & $\mathrm{H}_{3} \mathrm{PO}_{4}$ & $4,23 \pm 0,74$ & $29,47 \pm 6,11$ & $4,10 \pm 0,01$ & 49,76 \\
\cline { 2 - 6 } & $\mathrm{HCL}^{\mid}$ & $5,53 \pm 0,77$ & $27,43 \pm 6,70$ & $3,43 \pm 0,30$ & 47,60 \\
\cline { 2 - 6 } & $\mathrm{HNO}_{3}$ & $7,25 \pm 0,45$ & $22,49 \pm 0,34$ & $3,83 \pm 0,21$ & 55,60 \\
\cline { 2 - 6 } & $\mathrm{H}_{2} \mathrm{SO}_{4}$ & $12,29 \pm 1,04$ & $23,72 \pm 0,77$ & $4,86 \pm 0,06$ & 57,03 \\
\hline
\end{tabular}

Tabela 4 - Resultados do pré-tratamento para 1,0\% de ácido e 30 minutos.

\begin{tabular}{|c|c|c|c|c|c|}
\hline Biomassa & Ácido & AR & ART & BRIX & Rend \% \\
\hline \multirow{4}{*}{ NP } & $\mathrm{H}_{3} \mathrm{PO}_{4}$ & $3,18 \pm 0,87$ & $18,18 \pm 7,15$ & $4,36 \pm 0,55$ & 53,66 \\
\cline { 2 - 6 } & $\mathrm{HCL}_{4}$ & $3,88 \pm 0,84$ & $24,46 \pm 1,49$ & $3,83 \pm 0,66$ & 62,33 \\
\cline { 2 - 6 } & $\mathrm{HNO}_{3}$ & $12,42 \pm 1,04$ & $31,18 \pm 3,81$ & $4,43 \pm 0,21$ & 58,66 \\
\cline { 2 - 6 } & $\mathrm{H}_{2} \mathrm{SO}_{4}$ & $15,58 \pm 3,92$ & $26,99 \pm 1,14$ & $5,60 \pm 0,53$ & 46,66 \\
\cline { 2 - 6 } & \multicolumn{5}{|c}{} \\
\hline \multirow{4}{*}{$\mathbf{O P}$} & $\mathrm{H}_{3} \mathrm{PO}_{4}$ & $5,93 \pm 0,85$ & $22,23 \pm 6,45$ & $4,26 \pm 0,11$ & 59,66 \\
\cline { 2 - 6 } & $\mathrm{HCL}^{\mid}$ & $3,05 \pm 1,02$ & $27,03 \pm 1,52$ & $3,40 \pm 0,17$ & 67,50 \\
\cline { 2 - 6 } & $\mathrm{HNO}_{3}$ & $11,96 \pm 0,54$ & $24,17 \pm 2,44$ & $3,90 \pm 0,10$ & 53,66 \\
\cline { 2 - 6 } & $\mathrm{H}_{2} \mathrm{SO}_{4}$ & $10,20 \pm 1,96$ & $29,20 \pm 10,23$ & $4,66 \pm 0,25$ & 55,00 \\
\hline
\end{tabular}

Na Tabela 4 pode-se perceber que aumentando o tempo do processo de pré-tratamento a diferença entre os valores de AR, ART e Brix para os diferentes tipos de ácido mostrou que os melhores valores foram para ácido sulfúrico e nítrico. Comparando-se a Tabela 3 com a Tabela 4, observa-se que o aumento do tempo para o pré-tratamento não apresentou aumento significativo na quantidade de ART liberada no meio independente do tipo de ácido utilizado, mesmo que os valores de AR estejam maiores para 30 minutos do que em 15 minutos. A Figura 1 mostra os valores de ART para as duas espécies de palma estudadas para cada condição de tempo e concentração de ácido quando se utilizou o ácido sulfúrico. Observando a Figura 1 pode-se perceber que não houve variação significativa nos valores de ART quando se comparam as duas espécies de palma para nenhuma condição estudada. A melhor condição de pré-tratamento foi de 0,5\% de ácido sulfúrico em 15 minutos de reação. 


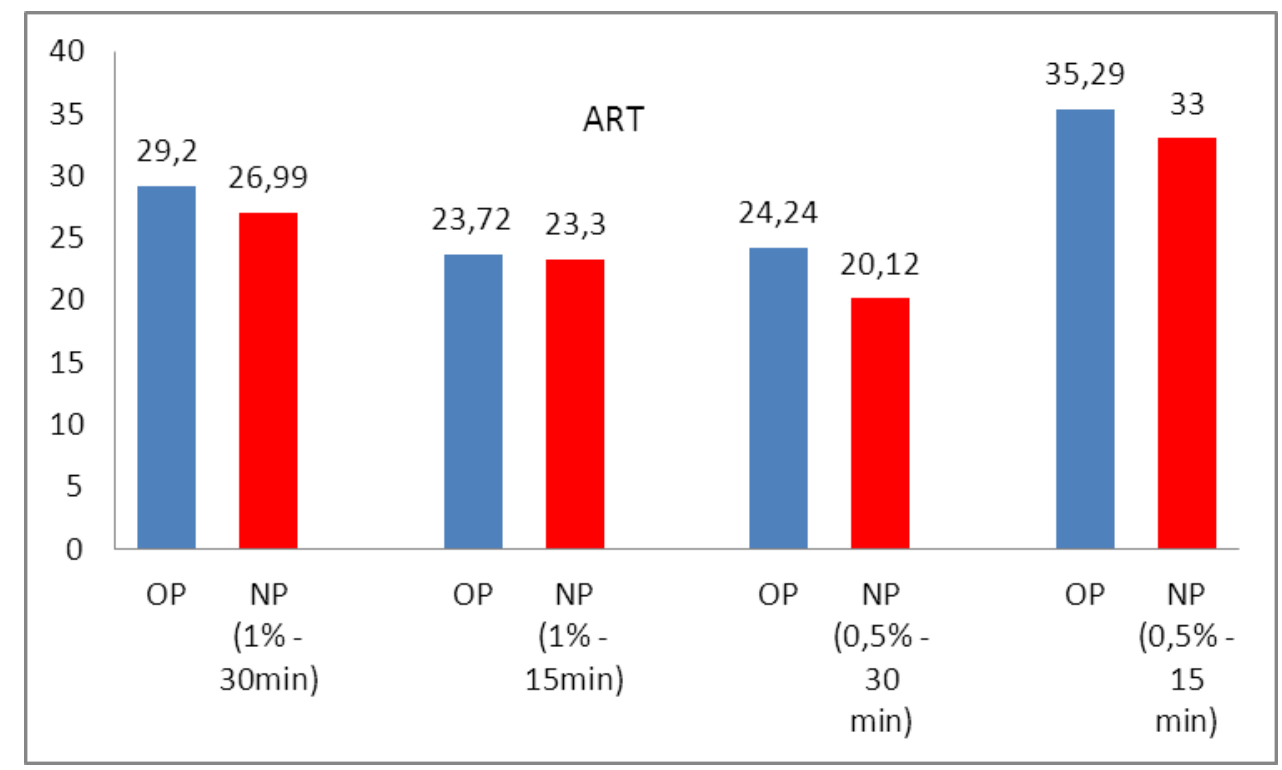

Figura 1 - Valores de ART para as espécies de palma utilizando ácido sulfúrico no prétratamento.

\section{CONCLUSÕES}

Analisando as tabelas, observa-se que as mudanças de tempo e da concentração de ácido propicia um aumento no somatório das concentrações de AR, ART e BRIX no pré-tratamento. Operando-se nesse estudo com diferentes tipos de ácido no pré-tratamento da biomassa também foi possível representar valores significativos de AR, ART, BRIX, indicando o ácido sulfúrico como o mais indicado em comparação aos outros ácidos utilizados. Dessa maneira, os dados apresentados demonstram que o pré-tratamento utilizado é capaz de desestruturar a parede vegetal da biomassa, em especial, celulose, hemicelulose e lignina, tornando os açúcares fermentescíveis disponíveis no processo de fermentação aos microrganismos para a conversão dos açúcares em etanol. Devido aos possíveis inibidores gerados no pré-tratamento, as melhores condições para esse processo devem ser analisadas após as etapas de hidrólise e fermentação.

\section{Agradecimentos}

Ao Conselho Nacional de Desenvolvimento Científico e Tecnológico - CNPq Processo No. 552595/2011-0 e Processo No. 420237/2013-5 pelos auxílios financeiros e bolsa PIBIC e ITI.

\section{REFERÊNCIAS}

CHEN, M.; XIA, L.; XUE, P. Enzymatic hydrolysis of corncob and ethanol production from cellulosic hydrolysate. International Biodeterioration and Biodegradation, Elsevier. 59 (2007) 85-89, 2006; 
LIRA, M. A.; SANTOS, M.V.F.; CUNHA, M.V.; MELLO, A.C.L.; FARIAS, I.; SANTOS, D.C. A Palma Forrageira na Pecuária do Semi-árido. In: GOMIDE, C.A.M. et al. Alternativas Alimentares para Ruminantes. Aracaju: Embrapa Tabuleiros Costeiros, 2006. cap.1, p. 17-33.

NASCIMENTO, V. M. Pré-tratamento alcalino (naoh) do bagaço de cana-de-açúcar para a produção de etanol e obtenção de xilooligômeros. Dissertação (Mestrado em Engenharia Química) - Centro de Ciências Exatas e Tecnologia, Departamento de engenharia química, Universidade Federal de São Carlos, 2011.

MENEZES, R. S. C.; SIMÕES, D. A.; SAMPAIO, E. V. S. B. A palma no Nordeste do Brasil Conhecimento atual e novas perspectivas de uso. Recife: Ed. Universitária da UFRPE, 2005, v. único, p. 44. 2005.

SÁNCHEZ, J; SÁNCHEZ, F; CURT, M. D; FERNÁNDEZ, J. Assessment of the bioethanol potential of prickly pear (Opuntia ficus-indica (L.) Mill.) biomass obtained from regular crops in the province of Almeria (SE Spain). Departamento de Producción Vegetal: Botánica y Protección Vegetal, Universidad Politécnica de Madrid (UPM), Madrid, Spain. Israel Journal of Plant Sciences, 2012.

SANTOS, M. V. F.; CUNHA, M. V. C.; LIRA, M. A.; DUBEUX Jr., J. C. B.; FREIRE, J. L.; PINTO, M. S. C.; SANTOS, D. C.; SOUZA, T. C.; SILVA, M.C.; Manejo da palma forrageira. $2^{\circ}$ congresso brasileiro de palma e outras cactáceas; Garanhuns, Recife, 2011.

SANTOS, M V. F.; LIRA, M. A.; DUBEUX JR, J.C.B. et al. Palma forrageira In: Plantas forrageiras.1 ed., Viçosa: Editora UFV, v.unico, p. 459-493. 2010.

SANTOS, M. S. R. Estudo de pré - tratamentos de palha e sabugo de milho visando a produção de etanol 2G. Dissertação de mestrado. Pós-graduação em Engenharia Química, Universdade Federal de Alagoas, Maceió-AL, 2014.

TAMANI, C; HAULY, M. C. O. Resíduos agroindustriais para produção biotecnológica de xilitol. Ciências Agrárias, Londrina, v. 25, n. 4, p. 315-330, out/dez. 2004.

TOSTO, M. S. L.; ARAÚJO, G. G. L.; OLIVEIRA, R. L.; BAGALDO, A. R.; DANTAS, F. R.; MENEZES, D. R.; CHAGAS, E. C. O. Composição química e estimativa de energia da palma forrageira e do resíduo desidratado de vitivinícolas. Rev. Bras. Saúde Prod. An., v.8, n.3, p. 239-249, jul/set, 2007.

WOLF, L. D. Pré-tratamento organossolve do bagaço de cana-de-açúcar para a produção de etanol e obtenção de Xilooligômeros. Dissertação (Mestrado em engenharia química) - Centro de Ciências Exatas e de Tecnologia Departamento de Engenharia Química, Universidade Federal de São Carlos, 2011. 